\title{
Quantum Turbulence Decay
}

\author{
Demosthenes Kivotides \\ Low Temperature Laboratory, Helsinki University of Technology, \\ P.O. Box 2200, FIN-02015 HUT, Finland
}

(Dated: November 21, 2018)

\begin{abstract}
We develop a computational model of quantum turbulence decay employing a kinematic prescription for the normal fluid. We find that after an initial transient, the length of the vortex tangle $L$ decreases and for large times obeys the scaling law $L \approx t^{-0.45}$. The average magnitude (along the quantized vortices) of the superfluid and line-vortex velocity are close and differ significantly from the average magnitude of the normal fluid velocity.
\end{abstract}

PACS numbers: $67.40 . V s, 47.27 . A k, 47.27 . G s$ 
In quantum turbulence physics [1], a tangle of quantized vortices interacts via mutual friction forces with thermal excitations (normal fluid) of the superfluid ground state. An elementary research program for this multifaceted problem investigates idealized flows characterized by symmetries like homogeneity in space and/or time, as well as, isotropy. At first, phenomenological issues like scalings of energy spectra [2, 3], energy decay [4] and structure functions should be established. This Letter contributes to this research program by employing a mathematical model of decaying quantum turbulence under conditions resembling the experiment of [4] and solving it with numerical and computational methods.

Our model consists of a dynamic equation describing the superfluid vortices and a kinematic prescription for the turbulent velocity field. In particular, if $\boldsymbol{S}(\xi, t)$ is the three dimensional representation of the vortex tangle then its motion obeys the equation [5]:

$$
\begin{aligned}
\frac{d \boldsymbol{S}}{d t}=\boldsymbol{V}_{\boldsymbol{l}}= & h \boldsymbol{V}_{\boldsymbol{s}}+h_{\times} \boldsymbol{S}^{\prime} \times\left(\boldsymbol{V}_{\boldsymbol{n}}-\boldsymbol{V}_{\boldsymbol{s}}\right)- \\
& h_{\times \times} \boldsymbol{S}^{\prime} \times\left(\boldsymbol{S}^{\prime} \times \boldsymbol{V}_{\boldsymbol{n}}\right)
\end{aligned}
$$

where the superfluid velocity $\boldsymbol{V}_{\boldsymbol{s}}$ is given by the Biot-Savart integral:

$$
\boldsymbol{V}_{\boldsymbol{s}}(\boldsymbol{x})=\frac{\kappa}{4 \pi} \int \frac{(\boldsymbol{S}-\boldsymbol{x}) \times d \boldsymbol{S}}{|\boldsymbol{S}-\boldsymbol{x}|^{3}}
$$

where $t$ is time, $\boldsymbol{x}$ is space, $\kappa$ is the quantum of circulation, $\boldsymbol{V}_{\boldsymbol{n}}$ is the velocity of the normal fluid, $\xi$ is the arclength along the loops, $\boldsymbol{S}^{\prime}=\frac{d \boldsymbol{S}}{\|d \boldsymbol{s}\|}$ is the unit tangent vector while $h, h_{\times}$and $h_{\times \times}$are constants related to mutual friction physics.

At every instant, the normal velocity is decomposed into a mean value and a fluctuation $\boldsymbol{V}_{\boldsymbol{n}}=\left\langle\boldsymbol{V}_{\boldsymbol{n}}\right\rangle+\boldsymbol{u}_{\boldsymbol{n}}$ with the fluctuation $\boldsymbol{u}_{\boldsymbol{n}}$ defined by the following function [6]:

$$
\begin{aligned}
\boldsymbol{u}_{\boldsymbol{n}}= & \sum_{m=1}^{M}\left[\boldsymbol{A}_{\boldsymbol{m}} \times \hat{\boldsymbol{k}}_{\boldsymbol{m}} \cos \left(\boldsymbol{k}_{\boldsymbol{m}} \cdot \boldsymbol{x}+\omega_{m} t\right)+\right. \\
& \left.\boldsymbol{B}_{\boldsymbol{m}} \times \hat{\boldsymbol{k}}_{\boldsymbol{m}} \sin \left(\boldsymbol{k}_{\boldsymbol{m}} \cdot \boldsymbol{x}+\omega_{m} t\right)\right]
\end{aligned}
$$

where $M$ is the number of wavemodes the sum of which constitutes the velocity field. $\boldsymbol{A}_{\boldsymbol{m}}$, $\boldsymbol{B}_{\boldsymbol{m}}$ are vectors with random orientation and magnitude $\left|\boldsymbol{A}_{\boldsymbol{m}}\right|^{2}=\left|\boldsymbol{B}_{\boldsymbol{m}}\right|^{2}=(2 / 3) E_{n}\left(k_{m}\right) \Delta k_{m}$ with $E_{n}\left(k_{m}\right)$ the normal fluid energy spectrum at wavenumber $k_{m}$. In addition, $\hat{\boldsymbol{k}}_{\boldsymbol{m}}$ is a unit vector normal to both $\boldsymbol{A}_{\boldsymbol{m}}$ and $\boldsymbol{B}_{\boldsymbol{m}}$ and $\boldsymbol{k}_{\boldsymbol{m}}=k_{m} \hat{\boldsymbol{k}}_{\boldsymbol{m}}$. The frequencies $\omega_{m}=\sqrt{k_{m}^{3} E_{n}\left(k_{m}\right)}$ correspond to the physical notion of "eddy turnover time". As required, $\boldsymbol{u}_{\boldsymbol{n}}$ is incompressible 

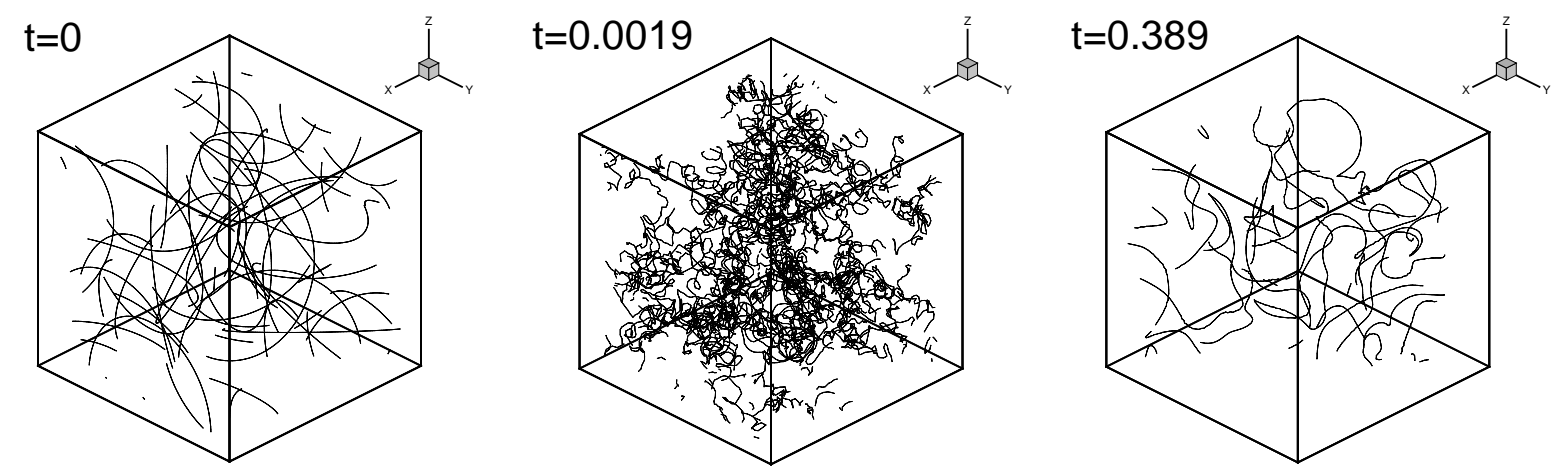

FIG. 1: Vortex tangle at initial, maximum length and stoppage times. To serve clarity, only one eighth of the computational box is shown.

by construction. The energy spectrum $E_{n}\left(k_{m}\right)$ has two parts: A high wavenumber part ("inertial range"): $E_{n}\left(k_{m}\right)=C_{K} \epsilon^{\frac{2}{3}} k_{m}^{-\frac{5}{3}}$, where $C_{K}=1.5$ is the Kolmogorov constant and $\epsilon$ is the rate of energy dissipation and a low wavenumber part ("large eddies"): $E_{n}\left(k_{m}\right)=A k_{m}^{2}$, where $A$ is defined by matching the two spectra at $k_{e}$ the "integral length scale" wavenumber. According to the permanence of large eddies hypothesis, $A$ remains constant during the decay of turbulence. We observe that in the present definition of $\boldsymbol{u}_{\boldsymbol{n}}$ there is no coupling between different modes and so there can be no energy flux in wavenumber space. This is in contrast to what happens in Navier-Stokes turbulence. In addition, the model is insufficient for capturing fine normal fluid effects like intermittency. On the other hand, it reproduces adequately a number of turbulence phenomenologies that relate to even order statistics like for example the Kolmogorov scaling or the Lagrangian flatness factor [7]. Moreover, since the model was devised having homogeneous, isotropic turbulence in mind, it is suitable for the description of the grid normal fluid turbulence in the [4] experiment.

We use the symbol $l_{e}$ for the integral scale of turbulence (peak of the spectrum) and the symbol $u_{n}^{\prime}$ for the turbulence intensity. It is $3 u_{n}^{\prime 2}=\sum_{i=1}^{3}\left\langle u_{n}^{i} u_{n}^{i}\right\rangle=2 E$, where $E$ is the kinetic energy of turbulent fluctuations. Knowledge of $l_{e}$ and $u_{n}^{\prime}$ at each time step allows the construction of the normal velocity field. In particular, we can calculate the turbulent Reynolds $R e_{t}=u_{n}^{\prime} l_{e} / \nu$ (where $\nu$ is the coefficient of viscosity), the energy dissipation rate $\epsilon=u_{n}^{\prime 3} / l_{e}$ and the Kolmogorov scale $\eta=l_{e} / R e_{t}^{3 / 4}$. We employ the decay model of [4] in order to calculate $l_{e}$ and $u_{n}^{\prime}$ as functions of time. According to this model, there are two periods of turbulence decay: During the first, $l_{e}$ is smaller than $l_{b}$ (the box size). During 
the second (which starts at $t_{s}=\frac{11}{5(2 \pi)^{\frac{5}{2}}} \sqrt{\frac{C_{K}^{3}}{A}}\left(l_{b}^{5 / 2}-l_{e 0}^{5 / 2}\right)$ with $l_{e 0}$ being the integral scale at $t=0), l_{e}$ remains constant and equal to $l_{b}$. The change in $l_{e}$ before its saturation is given by: $l_{e}(t)=2 \pi\left((5 / 11) \sqrt{\frac{A}{C_{K}^{3}}}\left(t+t_{0}\right)\right)^{2 / 5}$, with $t_{0}=\frac{11}{5(2 \pi)^{\frac{5}{2}}} \sqrt{\frac{C_{K}^{3}}{A}} l_{e 0}^{5 / 2}$. For times smaller than the $l_{e}$ saturation time the kinetic energy of turbulence $E$ is calculated from: $E(t)=E_{0}\left(1+\frac{t}{t_{0}}\right)^{-6 / 5}$, where $E_{0}=(9 / 6)(2 \pi)^{3} A / l_{e 0}^{3}$. We have found that the prefactor $9 / 6$ is necessary in order not to have a discontinuity in normal fluid energy at $t_{s}$. This condition is not satisfied by the prefactor $11 / 6$ in formula (4) of [4]. For post-saturation times it is: $E(t)=\frac{27 C_{K}^{3} l_{b}^{2}}{2(2 \pi)^{2}}\left(t+t_{0}+\right.$ $\left.t_{1}\right)^{-2}$, with $t_{1}=(4 / 5)(2 \pi)^{-5 / 2} C_{K}^{3 / 2} A^{-1 / 2} l_{b}^{5 / 2}$. The constants $t_{0}$ and $t_{1}$ define the virtual origin time $t_{v o}=-\left(t_{0}-t_{1}\right)$. As defined in [8] $t_{v o}$ is the time when (supposedly) the turbulence has infinite energy concentrated on an integral length scale of infinite wavenumber. In this interpretation as the energy decays the energy containing wavenumber moves towards smaller values. Our initial conditions correspond to an intermediate turbulence state in the decay process. We employ periodic boundary conditions for the superfluid tangle by introducing image vortices. The normal flow is periodic by construction.

The working fluid is ${ }^{4} \mathrm{He}-I I$ and so the quantum of circulation has the value $\kappa=9.97$. $10^{-4} \mathrm{~cm}^{2} / \mathrm{s}$. The calculation is done at $T=1.3 \mathrm{~K}$ (compared with $T=1.5 \mathrm{~K}$ in [4]) for which the other parameters of the problem have the values: $\nu=23.30 \cdot 10^{-4} \mathrm{~cm}^{2} / \mathrm{s}, h=0.978$, $h_{\times}=4.0937 \cdot 10^{-2}$ and $h_{\times \times}=2.175 \cdot 10^{-2}$. In addition, we have $R e_{t}=5 \cdot 10^{3}$ and the initial peak of the spectrum is located at $l_{e 0}=0.0161 \mathrm{~cm}$ which corresponds to $k_{e 0} \approx 62 \mathrm{~cm}^{-1}$. For comparison, $l_{b}=0.1 \mathrm{~cm}$ and $k_{b}=10 \mathrm{~cm}^{-1}$. We mesh the line vortices with discretization length $\Delta x=l_{b} / 84=1.19 \cdot 10^{-3} \mathrm{~cm}$. We use the same distance to define the smallest resolvable wavelength in the normal fluid turbulence model (eq. (3)), $l_{\text {co }}=2.381 \cdot 10^{-3} \mathrm{~cm}$ which corresponds to wavenumber $k_{c o}=420 \mathrm{~cm}^{-1}$. Using the equations of the model we can calculate the saturation time $t_{s}=0.2048 \cdot 10^{-2} s$, as well as, the time $t_{\eta r}$ at which $\eta$ will become equal to the smallest resolvable scale $l_{c o}: t_{\eta r} \approx 0.025 \mathrm{~s}$. Beyond this time the line vortices could develop structure at space scales smaller than the smallest normal turbulence wavelength. At stoppage time $t_{e}=0.389 \mathrm{~s}$, the Kolmogorov scale is equal to $5.26 \cdot 10^{-3} \mathrm{~cm}$ and therefore it is greater than the smallest resolvable wavelength equal to $2 \cdot \Delta x=2.38 \cdot 10^{-3} \mathrm{~cm}$. The stoppage time Kolmogorov scale corresponds to wavenumber $k_{\eta e}=190 \mathrm{~cm}^{-1}$. The time step is chosen in order to ensure that none of the (resolvable by the numerical grid) Kelvin waves propagates more than one discretization vortex segment within one calculation step. 
The typical time step for this is $\Delta t \approx 0.3 \cdot 10^{-3} s$. Initially, the tangle consists of 114 vortex rings of random orientation and its total length is $L_{0}=14.06 \mathrm{~cm}$. The choice of random initial conditions is justified for two reasons: (a) there is no experimental information about the actual initial tangle configuration which could be employed, (b) since the experimental scalings are reproducable without an explicit control over the geometry of the initial vortices it must be the case that the scaling phenomenology does not depend on the latter geometry. The second point agrees with our conception of turbulence as a statistical flow state that can be achieved from a variety of initial conditions. This is equivalent to our understanding of turbulence properties as idiosyncratic of the differential equations governing the system and not of the initial conditions. Different initial conditions are driven by the system to generic (reproducable) turbulence scalings.

Other useful quantities one can calculate are the average values of the velocity magnitudes $\left|\boldsymbol{V}_{\boldsymbol{l}}\right|,\left|\boldsymbol{V}_{\boldsymbol{n}}\right|,\left|\boldsymbol{V}_{\boldsymbol{s}}\right|$ and $|\cos (\theta)|=\left|\boldsymbol{S}^{\prime} \cdot\left(\boldsymbol{V}_{\boldsymbol{n}}-\boldsymbol{V}_{\boldsymbol{l}}\right)\right| /\left|\boldsymbol{V}_{\boldsymbol{n}}-\boldsymbol{V}_{\boldsymbol{l}}\right|$ along the vortex filaments at various times during the system's evolution. These averages are taken by sampling the quantities of interest at each discretization node, and subsequently forming their arithmetic mean. Angle $\theta$ is an important quantity in the physics of the mutual friction force per unit length: $\boldsymbol{f}=\rho_{s} \kappa d_{\times \times} \boldsymbol{S}^{\prime} \times\left(\boldsymbol{S}^{\prime} \times\left(\boldsymbol{V}_{\boldsymbol{n}}-\boldsymbol{V}_{\boldsymbol{l}}\right)\right)-\rho_{s} \kappa d_{\times} \boldsymbol{S}^{\prime} \times\left(\boldsymbol{V}_{\boldsymbol{n}}-\boldsymbol{V}_{\boldsymbol{l}}\right)$. Here $d_{\times}=-2.045 \cdot 10^{-2}$ and $d_{\times \times}=4.270 \cdot 10^{-2}$ are nondimensional coefficients and $\rho_{s}=138.6 \cdot 10^{-3} \mathrm{~g} / \mathrm{cm}^{-3}$ is the superfluid density.

The results $\left(\left\langle\boldsymbol{V}_{\boldsymbol{n}}\right\rangle=\mathbf{0}\right)$ show that in accordance to an instability discovered by Cheng et al [9] and elaborated mathematically by Glaberson et al 10], the normal flow excites Kelvin waves on the filaments (Fig[1). The length of the tangle reaches a maximum of $L_{\max }=47.83 \mathrm{~cm}$ at $t=0.0019 \mathrm{~s}$ and subsequently decreases. At maximum length the vortex line density is $\Lambda=L / V \approx 0.5 \cdot 10^{5}$ compared with $\Lambda \approx 2 \cdot 10^{5}$ for the smallest $\Lambda$ run in [4]. In stating this, we made use of the relation $\omega(t)=\kappa \Lambda$ in order to deduce $\Lambda$ from their superfluid vorticity $(\omega(t))$ data. Refering again to the smallest $\Lambda$ run in [4], we note that $\Lambda$ varies there over 2 orders of magnitude while here only by a factor of 5 . As required (Fig 2, right), the decay of turbulent normal fluid energy obeys the two previously mentioned temporal scaling laws. Also demonstrated in the same figure (left) are the two spatial scaling regimes in the $E_{n}(k)$ spectrum before $t_{s}$, as well as, the disappearance of the large eddies scaling regime for subsequent times. One can ask a two fold question: (a) 

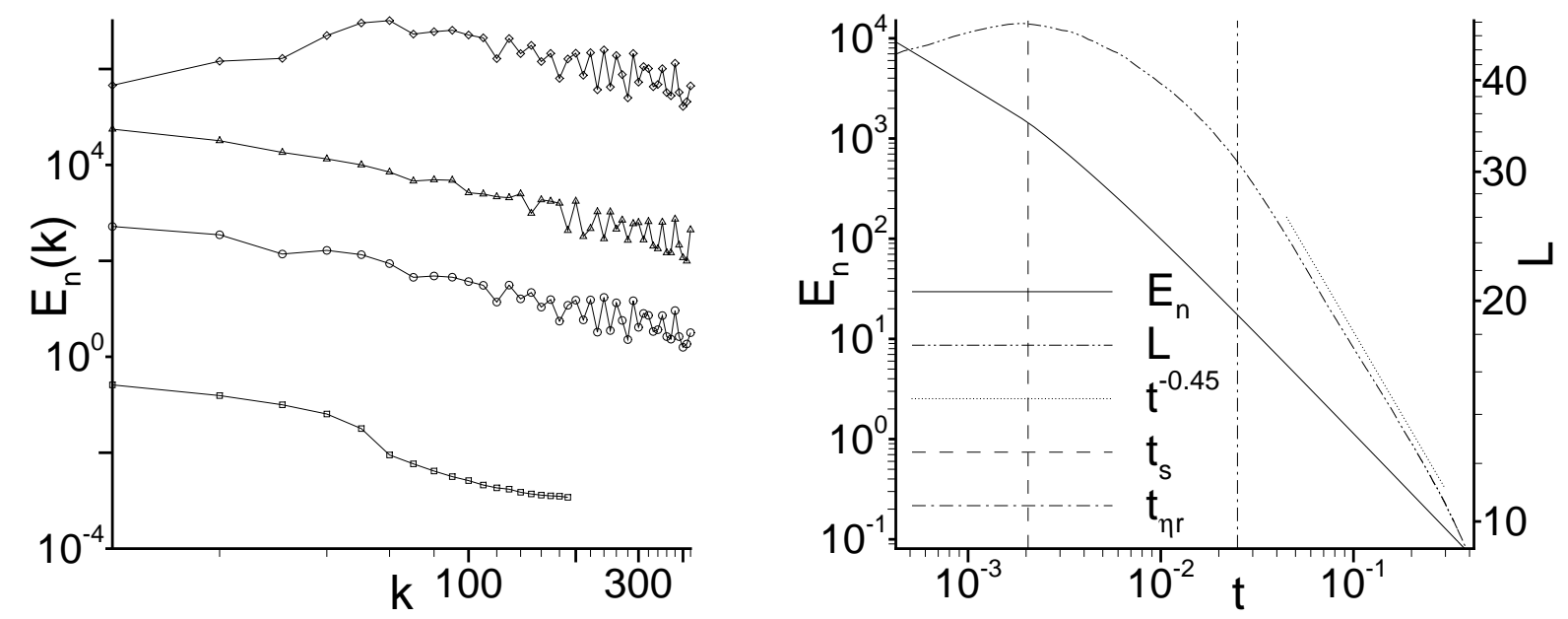

FIG. 2: Left: normal fluid spectra $E_{n}(k)$ at $t=0, t=t_{s}, t=t_{\eta r}$ and $t=t_{e}$. Right: the evolution of tangle length $L$ and normal fluid turbulent energy $E_{n}$.

why the vortex length decreases after a rapid transient and (b) why its observed temporal scaling at large times, $L \approx t^{-0.45}$, differs from the $L \approx t^{-1.5}$ one of $[4]$ ?

Possible reasons for the latter might be the inadequacy of the employed turbulence model or the shorter decay time span of 2 decades in the calculation compared to 3 in the experiment. In this milieu, an important question has to do with the meaning of the reported tangle lengths in both theory and experiment in the light of the findings of [11] that the superfluid tangle is a fractal. According to [12] (pg 25), the latter means that, as long as, the yardstick for length measurements belongs to the scale range within which the tangle satisfies a fractal scaling, different yardstick lengths will give a different length for the tangle. When it comes to calculation, this implies that better resolved fractal tangles would be measured to posses significantly greater lengths when the (different for each resolution) discretization length is used as yardstick length. Moreover, one could ask at first whether the second sound measurement technique introduces (in fractal turbulent tangles) such a yardstick length depending on the second sound wavelength/frequency. In other words, whether there exists a certain Kelvin wave frequency above which the experimentally employed second sound does not see the variations of the line density in a fractal vortex system. Subsequently, whether this possible uncertainty in the length measurement affects the scalings observed during the decay of turbulence. These matters are not clear-cut issues and deserve further 
investigation by expanding (for example) the work of [13] which calculated the motion of one roton shot toward a single straight line vortex in the realm of interactions between a roton and fractal vortex lines.

It is useful here to notice that in contrast to classical turbulence a uniform normal velocity field is not compatible with the assumption of isotropic superfluid turbulence and it is not dynamically irrelevant. These are clearly seen in counterflow quantum turbulence calculations [14] where the superfluid turbulence is due exclusively to a constant (externally imposed) velocity field. In these calculations, there is anisotropy in the direction of the imposed flow. The above are reminiscent of the role of uniform, imposed, magnetic fields in inducing anisotropies and affecting the velocity spectra in hydromagnetic turbulence (page 132 in [15] and page 100 in [16]). In order to clarify better this point we have done two more calculations, one with stationary turbulence and another with turbulence decay but with the addition of a constant normal velocity field in the $y$ direction. We have fixed the velocity magnitude $\left|\left\langle\boldsymbol{V}_{\boldsymbol{n}}\right\rangle\right|=V_{n y}=7 \mathrm{~cm} / \mathrm{s}$ so that it is comparable to the grid towing velocity (between 5 and $200 \mathrm{~cm} / \mathrm{s}$ ) in [4]. Although in this way $u_{n}^{\prime} / V_{n y} \approx 100$ (at $t=0$ ) one observes in Fig 3 (right) that with the imposed velocity field the vortex length increases at times for which (in the case of purely decaying turbulence) it decreases. Therefore, the temporal decay law for the line-vortex length could be affected by small bulk normal fluid velocities. We implicitly assume here that the mean velocity profile is stable; this is also the case in [14].

To analyze the first leg of the previously posed question, we note that according to the analysis of the [9, 10] instability (see also the discussion in 17]) the normal fluid velocity fluctuations transfer energy to a Kelvin wave of a particular wavelength (and therefore increase its amplitude) only when their component along the direction of motion of the wave is both greater in magnitude than the group velocity of the wave and parallel (of the same sign) to it. Any normal fluid velocity antiparallel to a vortex wave reduces its amplitude. Therefore since initially the turbulence intensity is approximately 100 times the group velocity of the fastest (resolvable) Kelvin wave and there are no Kelvin waves present, there is unhindered Kelvin wave excitation. This explains the initial rapid increase of vortex length. Subsequently, in conjunction with the decay of turbulence intensity, at places with wave group velocity (a) larger than the local normal fluid velocity magnitude or (b) antiparallel to the normal fluid velocity direction, the wave amplitudes are damped. The 


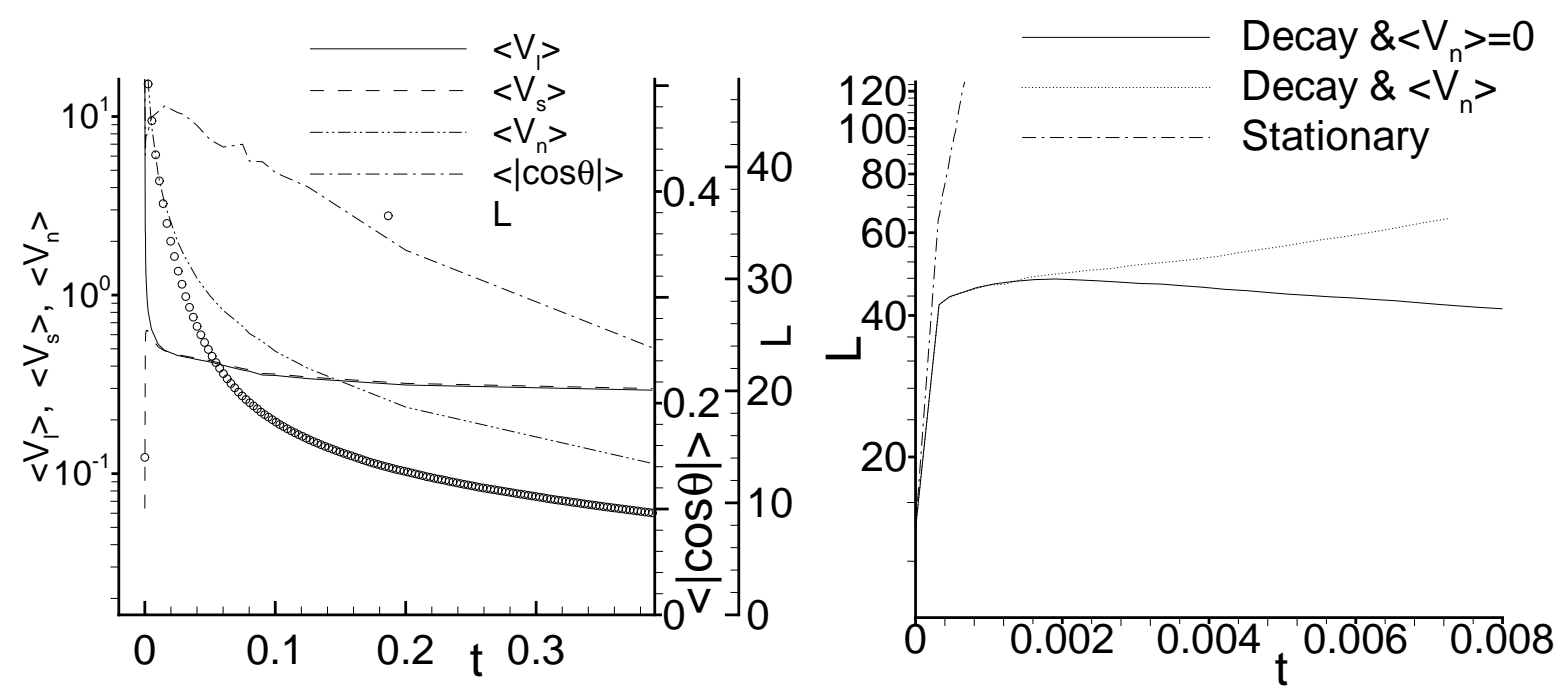

FIG. 3: Left: average values of $\left|\boldsymbol{V}_{\boldsymbol{l}}\right|,\left|\boldsymbol{V}_{\boldsymbol{n}}\right|,\left|\boldsymbol{V}_{\boldsymbol{s}}\right|$ and $|\cos (\theta)|$ along the quantized vortex filaments. Right: evolution of tangle length $L$ in three calculations with different normal fluid turbulence characteristics.

turbulence decay factor is necessary since as Fig 3 (right) shows, in stationary turbulence the length keeps increasing rapidly.

The results for the average values of $\left|\boldsymbol{V}_{\boldsymbol{l}}\right|,\left|\boldsymbol{V}_{\boldsymbol{n}}\right|,\left|\boldsymbol{V}_{\boldsymbol{s}}\right|$ and $|\cos (\theta)|$ are presented in Fig 3 (left). They show that the initial length transient corresponds to rapid $\left\langle\left|\boldsymbol{V}_{\boldsymbol{l}}\right|\right\rangle$ and $\left\langle\left|\boldsymbol{V}_{\boldsymbol{s}}\right|\right\rangle$ transients. The latter increases towards a maximum coinciding in time with the length maximum while the former decreases approaching $\left\langle\left|\boldsymbol{V}_{\boldsymbol{s}}\right|\right\rangle$. Notice that when the length starts decreasing there is order of magnitude difference between $\left\langle\left|\boldsymbol{V}_{\boldsymbol{n}}\right|\right\rangle$ and $\left\langle\left|\boldsymbol{V}_{\boldsymbol{s}}\right|\right\rangle$. This is particularly true for the average of $\left|\boldsymbol{V}_{\boldsymbol{s}}\right|$ taken over the whole volume of the fluid since this was found (using a $84^{3}$ grid) to be an order of magnitude smaller than the average over the line vortices. Another observation is that for $\left\langle\left|\boldsymbol{V}_{\boldsymbol{n}}\right|\right\rangle$ smaller than $\left\langle\left|\boldsymbol{V}_{\boldsymbol{s}}\right|\right\rangle$ (which happens at very small normal turbulence energies) $\boldsymbol{S}^{\prime}$ tends to become normal to $\boldsymbol{V}_{\boldsymbol{n}}-\boldsymbol{V}_{\boldsymbol{l}}$. The results bring forward quantum turbulence physics that differ significantly from those proposed in [4] where the assumption was made that the superfluid and normal fluid velocities are identical. However, one must also bear in mind that the employed turbulence model does not have the quality of fully dynamical Navier-Stokes calculations. The latter kind of computations would eventually be required in order to verify the present results.

Overall, it is not possible to argue for the quality of the established mathematical models 
in quantum turbulence theory, as long as, the available experimental data fail to address directly the main variables (fluid velocities, vortex tangle geometry) in these models. This is a major obstacle for progress in quantum turbulence research. The present work suggests that progress depends on the development of new, more potent experimental methods and their combination with fully dynamic mathematical calculations. The latter could identify generic and essential phenomenological trends that could be encoded in statistical mechanical equations.

This research was supported by the Commission of the European Union under Contract \# HPRI-CT-1999-00050. I thank Matti Krusius and Carlo Barenghi for discussions, as well as, Caltech for computing time.

[1] W. F. Vinen and J. J. Niemela, J. Low Temp. Phys. 128, 167 (2002).

[2] J. Maurer and P. Tabeling, Europhys. Lett. 43, 29 (1998).

[3] D. Kivotides, J. C. Vassilicos, D. C. Samuels, and C. F. Barenghi, Europhys. Lett. 57, 845 (2002).

[4] S. R. Stalp, L. Skrbek, and R. J. Donnelly, Phys. Rev. Lett. 82, 4831 (1999).

[5] O. C. Idowu, D. Kivotides, C. F. Barenghi, and D. C. Samuels, J. Low Temp. Physics 120, $269(2000)$.

[6] J. C. H. Fung and J. C. Vassilicos, Phys. Rev. E 57, 1677 (1998).

[7] N. A. Malik and J. C. Vassilicos, Phys. Fluids 11, 1572 (1999).

[8] L. Skrbek and S. R. Stalp, Phys. Fluids 12, 1997 (2000).

[9] D. K. Cheng, M. W. Cromar, and R. J. Donnelly, Phys. Rev. Lett. 31, 433 (1973).

[10] W. I. Glaberson, W. W. Johnson, and R. M. Ostermeier, Phys. Rev. Lett. 33, 1197 (1974).

[11] D. Kivotides, C. F. Barenghi, and D. C. Samuels, Phys. Rev. Lett. 87, 155301 (2001).

[12] B. B. Mandelbrot, The Fractal Geometry of Nature (Freeman, 1977).

[13] D. C. Samuels and R. J. Donnelly, Phys. Rev. Lett. 65, 187 (1990).

[14] K. W. Schwarz, Phys. Rev. B 38, 2398 (1988).

[15] J. R. Herring and J. C. McWilliams, eds., Lecture Notes on Turbulence (World Scientific, 1989).

[16] D. Biskamp, Magnetohydrodynamic Turbulence (Cambridge University Press, 2003). 
[17] D. C. Samuels and D. Kivotides, Phys. Rev. Lett. 83, 5306 (1999). 